\title{
Schritt für Schritt: die Geburt des modernen Schuhs
}

Nike U. Breyer

Gastkuratorin

der Sonderausstellung

nike.breyer[at]web.de

Schritt für Schritt. Die Geburt des modernen Schuhs

Ausstellung im Deutschen Medizinhistorischen Museum Ingolstadt, Anatomiestrasse 18-20, D-85049 Ingolstadt

Bis 14. Oktober 2012, Dienstag bis Sonntag 10 bis 17 Uhr, Eintritt 5 Euro, Katalog zur Ausstellung 8 Euro

Begleitprogramm über: www.dmm-ingolstadt.de weite zur Kenntnis genommen wurde dieser interessante Anpassungsprozess jedoch bisher kaum.

Dies will das Deutsche Medizinhistorische Museum Ingolstadt ändern und hat nun unter dem Titel «Schritt für Schritt. Die Geburt des modernen Schuhs» diesem spannenden Thema eine Mini-Ausstellung gewidmet. Das Thema «Schuhreform» ist dabei auch deshalb im Medizinhistorischen Museum gut aufgehoben, weil Schuhe und Medizin über die Konsequenzen von Ersteren für den Körper unmittelbar zusammengehören: Im Banne der Mode und anderer kultureller Codices, unterstreicht die Museumsleiterin Prof. Dr. Marion Maria Ruisinger, wurde allzu oft «die Fussbekleidung zu einem qualvollen und höchst «medizinischen〉 Problem».

Da passt es gut ins Konzept, dass der «Vater der Schuhreform», der durch seine Schriften diesen Reformprozess massgeblich angestossen hat, von Haus aus Anatom war: Georg Hermann von Meyer (18151892) (Abb. 1), ein gebürtiger Frankfurter, verbrachte und lehrte mit über vierzig Jahren die wesentliche Zeit seines Forscherlebens als Professor an der Universität in Zürich. «Die «Geburt des modernen Schuhs»», meint Ruisinger, «fand also, wenn man so (Repro, Quelle: Katalog)

will, in der Anatomie statt.»

Die Geschichte der Schuhreform wird in Ingolstadt in drei thematischen Modulen erzählt. Diese entsprechen den drei massgeblichen historischen Stationen und zeigen neben Schuhen, Leisten und Büchern auch schriftliche Originaldokumente und Fotos.

Das erste Modul beleuchtet als Ausgangspunkt das Thema «symmetrische Schuhe». Seit der Renaissance wird Symmetrie in der europäischen Kultur mit einer mathematisch gedachten höheren Ordnung in Verbindung gebracht und steht für die Idee von Vollkommenheit. Auch der Körper musste sich, zu sehen an den Schuhen, diesen Vorstellungen fügen. 
Das älteste Exponat der Ausstellung ist ein symmetrischer schwerer Reiterstiefel aus der Zeit um 1700 (Abb. 2). Bis auf das kolossale Gewicht dürfte dieser «Rohling» den Füssen allerdings noch kaum grössere Beschwerden zugefügt haben. Was nicht passte, wurde passend gemacht, mit hineingestopften Lappen, Stroh oder Werg (einem faserigen Arbeitsmaterial aus Hanf o.ä.). Dass die Schuhe entsprechend grösser waren, als es die Fussgrösse erfordert hätte, dürfte kaum aufgefallen sein. Das änderte sich, als die Schuhe im Rokoko, Empire und Biedermeier selbst für das Militär - aristokratisch schlank und körperbetonter gearbeitet wurden. Damit kam die anatomisch falsche, weil symmetrische Form erst

\section{«Der Chiropodist hobelte, schnitt und ätzte an Schwielen und Hühneraugen.»}

«richtig zum Tragen». Fussmalaisen nahmen dramatisch zu, für die im späten 18. Jahrhundert «Chiropodisten» mit Tinkturen und Skalpell ihre Dienste anboten. Wieder hiess es, was nicht passt, wird passend gemacht. Der Chiropodist hobelte, schnitt und ätzte an Schwielen und Hühneraugen, die der Fussbesitzer sich selbst eingehandelt hatte, indem er seinen Fuss in «schönen», aber unpassenden Schuhen gewaltsam modellierte. Kritik an diesen Schuhen und Praktiken äusserte als einer der Ersten 1781 der holländische Anatom Pieter Camper (1722-1789) in seiner «Abhandlung von der besten Form der Schuhe» - in Ingolstadt im Original zu sehen. Wirkung zeitigte diese moderate Missfallensbekundung eines Wissenschaftlers jedoch nicht - noch nicht. Die entschei-

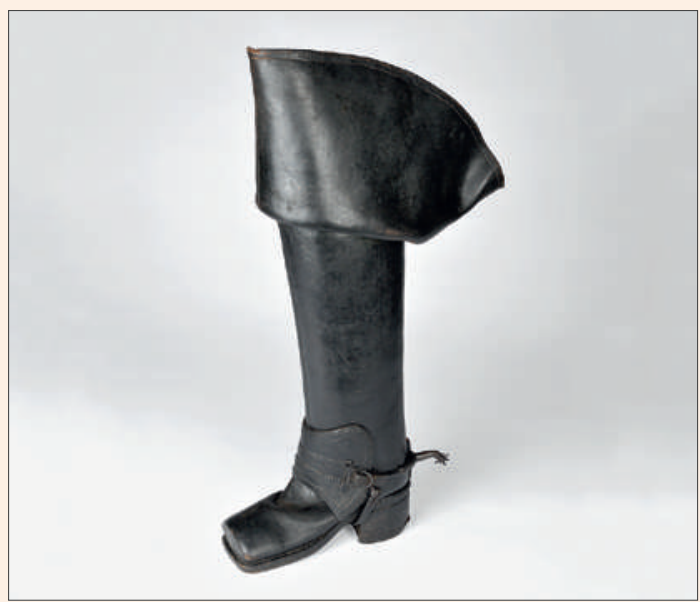

Abb. 2: Kavalleriestiefel mit Sporen. Schwarzes Leder, um 1700. Bayerisches Armeemuseum, Ingolstadt. dende Wende brachte erst 1858 ein kleines, deutlich energischer formuliertes «Manifest» mit dem Titel «Die richtige Gestalt der Schuhe. Eine Abhandlung aus der angewandten Anatomie für Ärzte und Laien geschrieben». Darin forderte der besagte Zürcher Anatomieprofessor Georg Hermann von Meyer ein Ende der in seinen Augen barbarischen Vergewaltigung der Füsse durch modisch-symmetrische Schuhe und die Ersetzung durch anatomiegerechtes Schuhwerk. Er stiess damit überraschend auf offene Ohren, zunächst beim schweizerischen Militär - was durch Aktennotizen, Korrespondenzen, Erlasse und ähnliche Unterlagen im Militärarchiv Bern gut dokumentiert ist [2] - bald aber auch bei anderen Militärverwaltungen Europas und ebenso in zivilen Kreisen (Abb. 3). In der Ausstellung ist eine Erstausgabe dieser kleinen Schrift zu sehen. Sie markiert den geistigen Knotenpunkt der gesamten Präsentation. Meyers neuartiges Verfahren der mathematischen Konstruktion einer anatomiegerechten Brandsohle, die sich von gängigen Schusterverfahren kategorial unterschied, wird in Ingolstadt durch Verweise auf andere Forschungen von ihm (seine bedeutende Entdeckung der Struktur der Substantia Spongiosa, der Knochenbälkchen) in einen plausiblen Zusammenhang gebracht.

Dass die Bälkchen im Knocheninneren der Gewichtsersparnis dienen, war damals bereits bekannt. Meyer zeigte nun, dass ihre Anordnung den Druckund Zugkräften unter Belastung entspricht. Damit konnte er die Struktur der Spongiosa beispielhaft als das Ergebnis einer funktionellen Anpassung erklären. Ideen, die auch einen indirekten Einfluss auf die Konstruktion des Eiffeltums gehabt haben dürften (Abb. 4).

Der schweizerische Hauptmann, Buchautor und leidenschaftliche Meyer-Anhänger Auguste Salquin gab der Schuhreform mit seinem Begriff des «rationellen Schuhwerks» den letzten terminologischen

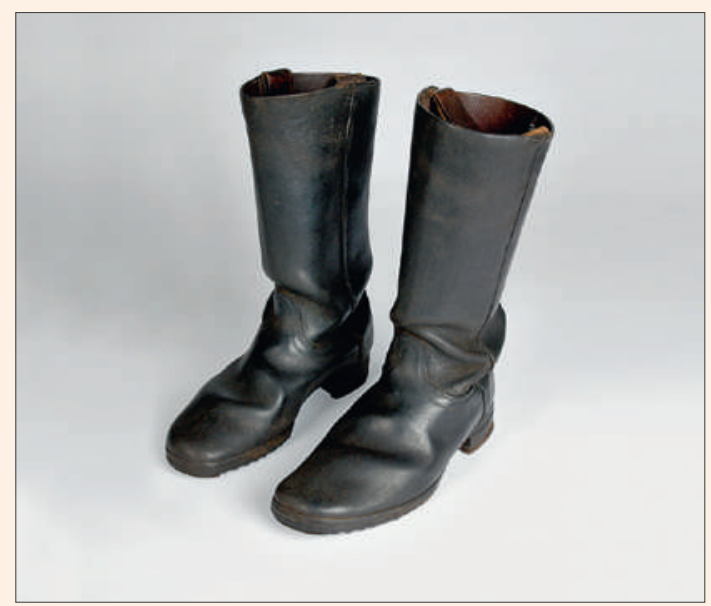

Abb. 3: «Knobelbecher» für Fusstruppen. Erster Weltkrieg Bayerisches Armeemuseum, Ingolstadt. Fotos: Michael Kowalski, Ingolstadt 
Abb. 4: Der Eiffelturm als Naturstruktur: 1867 hielt von Meyer vor der Naturforschenden Gesellschaft Zürich einen Vortrag über die «intelligente» Struktur der Spongiosa. Dabei ereignete sich eine Szene, die 1917 wie folgt geschildert wurde: «Als Zuhörer befand sich in dieser historisch denkwürdigen Sitzung [Karl] Eugen Culmann. Er sah sofort, dass die von Meyerschen Systeme genau den von der graphischen Statik, deren Begründer er war, konstruierten Kurven entsprechen, nach denen sich im Inneren von belasteten Trägern Druck und Zug verteilt [...]. Nach diesen Regeln hat ein Schüler Culmanns (Maurice Koechlin) den Plan zum Eiffelturm entworfen.» Koechlin verwendete dafür später nichts anderes als eine eiserne Spongiosa, die mit «einem Minimum von Material nur die mechanisch beanspruchten Linien aufführt und das Maximum von Tragfähigkeit erreicht.» $[3,4]$ (ETH-Bibliothek, Archive, Hs 1092: 1. Koechlin, Maurice: Pylône de 300 mètres de hauteur pour la ville de Paris 1889. Zeichnung, Paris 6.6.1884, Vorprojekt 6.6.1884 von E. Nouguier et M. Koechlin. Zeichnung 1:50 ausgeführt durch Maurice Koechlin, Paris, im Massstab 1:50, Bleistift, Farbe und Tusche, $75 \times 53 \mathrm{~cm}$.)

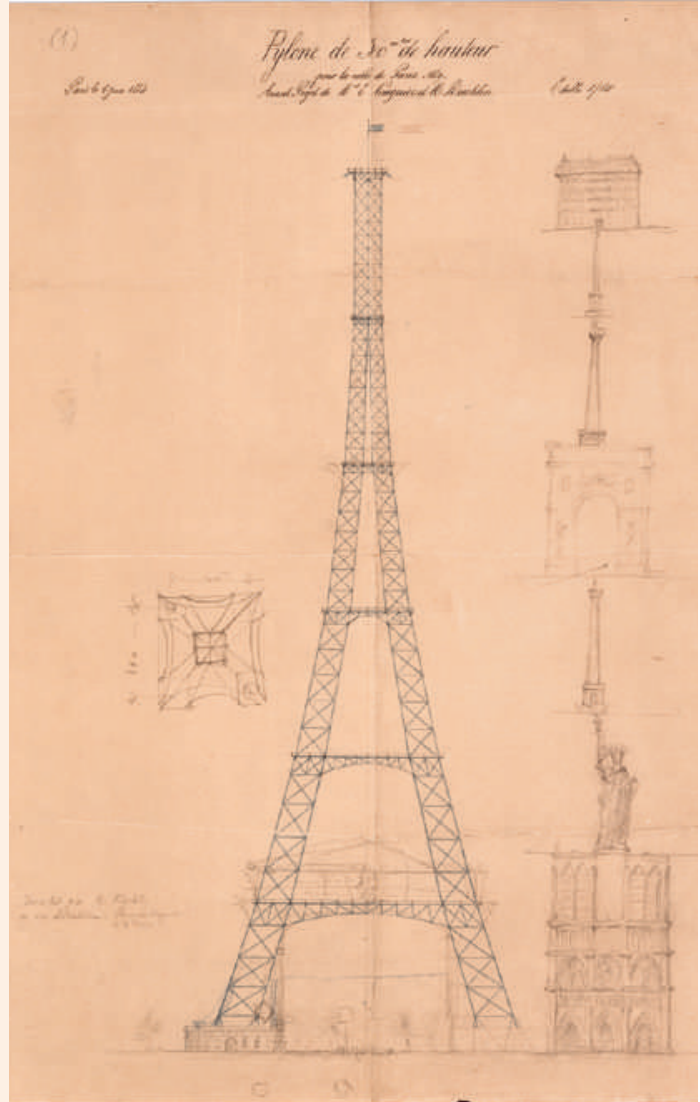

Schliff. Damit wurde der weitere Erfolg des neuen Schuhwerks erheblich befördert.

Im dritten Modul der Ausstellung und der chronologisch letzten Station der Schuhreform werden frühe Umsetzungsversuche der neuen Idee mit «Reformschuhen», Leisten und schriftlichen Dokumenten veranschaulicht und diese zugleich verschiedenen Personengruppen zugeordnet: Den Militärs lag die Marsch- und Kampffähigkeit ihrer Soldaten am Herzen («rationelle» Marschstiefel), Ärzten und Hygienikern die Gesunderhaltung der Bürger (medizinische

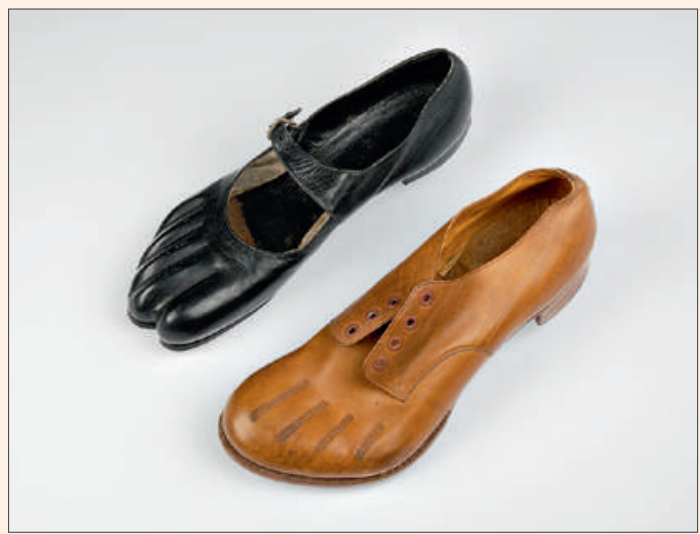

Abb. 5: Zehenkammerschuhe, um 1911. Nach einem Patent des späteren Stahlrohr-Industriellen Max Mannesmann von 1907. Salzgitter AG, Konzernarchiv/Mannesmann-Archiv, Mülheim a. d. Ruhr.

Foto: Michael Kowalski, Ingolstad
Literatur, u.a. mit dem neuen Argument des Röntgenbilds), den Schuhmachern das eigene Überleben gegen die Konkurrenz der Maschine (Schuhmacherlehrbücher) und den «Lebensreformern» die Befreiung des Körpers von den «Rohheiten der Mode» und einer auf Abwege geratenen «Civilisation» (Reformschuhe, Abb. 5, 6). Damit beleuchtet das dritte Ausstellungsmodul skizzenhaft, wie die Idee einer Schuhreform in ihrer Rezeption durch Partikularinteressen «überschrieben» und damit zugleich modifiziert wurde.

Jenseits aller vergänglichen Ideologeme, so zeigt sich aus der Distanz, hat sich die pragmatische, hygienisch-medizinisch begründete Variante einer neuen Fussbekleidung dauerhaft behauptet. Mit seiner Sohlenkonstruktion auf der Basis anatomischer Gesetze, auch wenn diese inzwischen korrigiert und verfeinert wurde, hat Georg Hermann von Meyer die Formgebung von Schuhen auf eine wissenschaftliche Basis gestellt. Diese wurde seither nicht mehr verlassen. Der moderne Schuh war geboren.

Dass wir alle scheinbar ganz selbstverständlich einen rechten und einen linken Schuh tragen, sieht man nach einem Besuch der Ausstellung im Medizinhistorischen Museum Ingolstadt - hoffentlich - mit anderen Augen.

\section{Literatur}

1 Beely F, Kirchhoff E. Der menschliche Fuss, seine Bekleidung und Pflege. Tübingen: Laupp; 1892.

2 Arni M. Es drückt der Schuh. Die Fussbekleidungsfrage in der Schweizer Armee 1850-1918. Schriftenreihe der Eidgenössischen Militärbibliothek.Nr. 43. Bern: Bibliothek am Guisanplatz; 2010.

3 Dem ersten Preisträger des Georg-Hermann-vonMeyer-Preises. Frankfurt/Main: Senckenbergische Naturforschende Gesellschaft; 1917.

4 http://blogs.ethz.ch/digital-collections/?s=eiffelturm

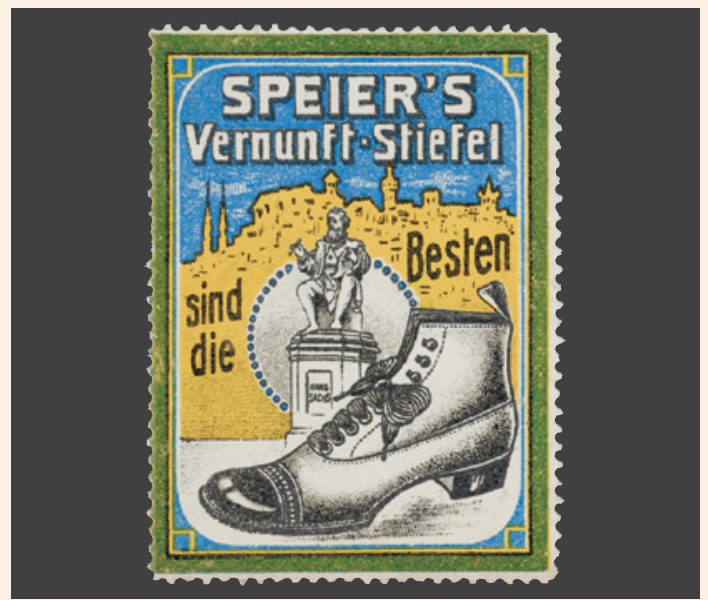

Abb. 6: Reklamemarke für städtisch-elegante Reformstiefel, Deutschland, frühes 20. Jahrhundert.

Quelle: Katalog 